\title{
ORIGINAL ARTICLE

EFFECTS OF POSTEROANTERIOR LUMBAR SPINE MOBILIZATIONS ON
PAIN, ROM AND FUNCTIONAL DISABILITY IN FEMALE SUBJECTS
WITH CHRONIC NONSPECIFIC LOW BACK PAIN

\author{
${ }^{1}$ L. V. S. Pravallika \\ ${ }^{2}$ C. Shanthi \\ ${ }^{3} \mathrm{~K}$. Madhavi
}

\section{ABSTRACT}

Background: Chronic nonspecific low back pain (CNSLBP) i.e., low back pain of at least 12 weeks duration without a specific cause is a major cause of activity limitation, absenteeism, and high health care expenses. The prevalence of CNSLBP is estimated approximately $23 \%$ and activity limitation due to LBP have been found to be $11 \%$ to $12 \%$ of the population. Previous studies comparing the efficacy of postero-anterior mobilisation and prone-press ups were done and revealed statistically significant improvements in extension ranges but not clinical relevant improvements. This is possibly attributable to single session of interventions. So long term gains in pain reduction and lumbar extension cannot be assumed. Hence this study would be intending to prove the effect of postero-anterior mobilisation and prone press ups on chronic non-specific low back pain after 6 weeks and their clinical application.

Method: 30 subjects who met the inclusion criteria were selected randomly from the department of physiotherapy, SVIMS and BIRRD, Tirupati. The study conducted for a period of 6 weeks.2 groups were formed with 15 in each group. PA lumbar glide and prone press up's was given to group I and only prone press up's was given to group II. Subjects were evaluated pre and post treatment for VAS, extension ROM of lumbar spine and functional disability.

Result: Results showed that there exists a statistical significance between the groups in all the 3 parameters. Present randomized clinical trial provided evidence to support the use of postero-anterior mobilisation and prone press-ups in relieving pain, improving ROM and reducing disability in subjects with non-specific low back pain. In addition, results supported that postero-anterior mobilisation was more effective than prone press-ups.

Keywords: Nonspecific low back pain, prone press up, postero anterior spinal mobilizations.

Received $10^{\text {th }}$ July 2014, revised $26^{\text {th }}$ August 2014, accepted $02^{\text {nd }}$ December 2014

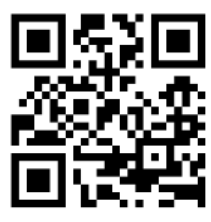

www.ijphy.org
DOI: 10.15621/ijphy/2014/v1i5/55272

\footnotetext{
${ }^{2}$ Assistant professor, College of Physiotherapy, SVIMS, Tirupati, India.

${ }^{3}$ Professor and Principal, College of

Physiotherapy, SVIMS, Tirupati, India.
}

\section{CORRESPONDING AUTHOR}

\section{${ }^{1}$ L. V. S. Pravallika}

MPT, College of Physiotherapy, SVIMS, Tirupati, India. 


\section{INTRODUCTION}

Chronic Nonspecific low back pain (CNSLBP) is defined as pain over the lower back not usually associated with any specific pathological structural changes with atleast 12 weeks of duration. ${ }^{1}$ People who report low back pain often have reduced spinal motion. When motion is limited, spinal extension is frequently more restricted than flexion. ${ }^{2}$ In India incidence of low back pain has been reported to be $23.09 \%$ and has lifetime prevalence of $60-85 \%$.low back pain affects men and women equally, with onset most often between the ages 30 to 50 years. ${ }^{3,4}$ Mechanical factors usually associated with initiation and exacerbation of chronic low back pain are sustained low load posture and movement, exposure to whole body vibration, repeated spinal loading has a negative impact on musculo-skeletal system. ${ }^{5}$ Common causes for nonspecific low back pain includes incorrect postures, instability, obesity, sedentary life style, muscle strain or ligamentous injury. Clinical Features include pain in lumbar region radiating to both the thighs but not below knees and relieved by rest. Pain exacerbated by activity and exercise. ${ }^{6}$ The conservative treatment of nonspecific low back pain includes electrotherapy, exercise therapy and manual therapy.

Maitland's concepts involve the application of passive and accessory oscillatory movements to spinal and vertebral joints to treat pain and stiffness of a mechanical nature. The technique aims to restore motions of spin, roll, glide between joint surfaces and are graded according to their amplitude. $^{7}$ PA central pressure is used in conditions of lumbar spine that cause pain which is evenly distributed to both the sides of body. This technique is of value in patients whose symptoms arise from that part of lumbar spine where there are structural changes associated with faulty posture. This technique is indicated when pain or spasm is felt on movement in this direction but it is performed in such a way that pain or spasm not provoked. ${ }^{8}$ McKenzie and May advocated a more general approach in which prone press -up exercise is used as a means of decreasing pain and increasing spinal motion. ${ }^{9}$ Hence, spinal mobility exercises should be recommended to low back patients. ${ }^{10}$ Basic philosophy of Mc Kenzie's theory is that the reverse forces can probably abolish the pain and restore function. Previous studies comparing the efficacy of postero-anterior mobilisation and prone-press ups were done after a single session and revealed statistically significant improvements in extension ranges but not clinical relevant improvements. This is possibly attributable to single session of interventions. So long term gains in pain reduction and lumbar extension cannot be assumed. Moreover only few studies have been done to see the effectiveness of postero-anterior spinal mobilisation on lumbar extension in non-specific low back pain and showed no specific clinical improvement. ${ }^{11}$

So there is lack of evidence for clinical effectiveness of postero-anterior spinal mobilisation, prone press-ups on lumbar extension in chronic non-specific low back pain. No study has been performed to see their effect after 6 weeks. Hence this study would be intending to prove the effect of postero-anterior mobilisation and prone press ups on non-specific low back pain after 6 weeks and their clinical application. Therefore, the objectives of this randomized controlled trial was to determine the effectiveness of the PA lumbar spine mobilizations in female subjects with chronic nonspecific low back pain for the outcomes of pain, ROM and functional disability.

\section{METHODOLOGY}

DESIGN OVERVIEW: This study was experimental study and samples were collected by simple random sampling.

SETTING AND PARTICIPANTS: Study was conducted in college of physiotherapy SVIMS and BIRRD Tirupati, between August 2013 to January 2014. Inclusion criteria: patients with chronic nonspecific low back pain (atleast 12 weeks duration), decreased lumbar extension, increased pain with lumbar extension during standing, Subjects willing to participate in the study, Females aged 35 to 50 years old. Exclusion criteria: Patients with Gross spinal deformity, Spinal instability/Hyper mobility, Spondylolisthesis, Fracture spine, Lumbar disk pathology, Prior low back surgery, Evidence of cord / root involvement, Cardiovascular disease, Uncontrolled hypertension, Severe respiratory disease, Urinary or faecal incontinence, Pregnancy, Hernia (Hiatal, Abdominal, Inguinal) 


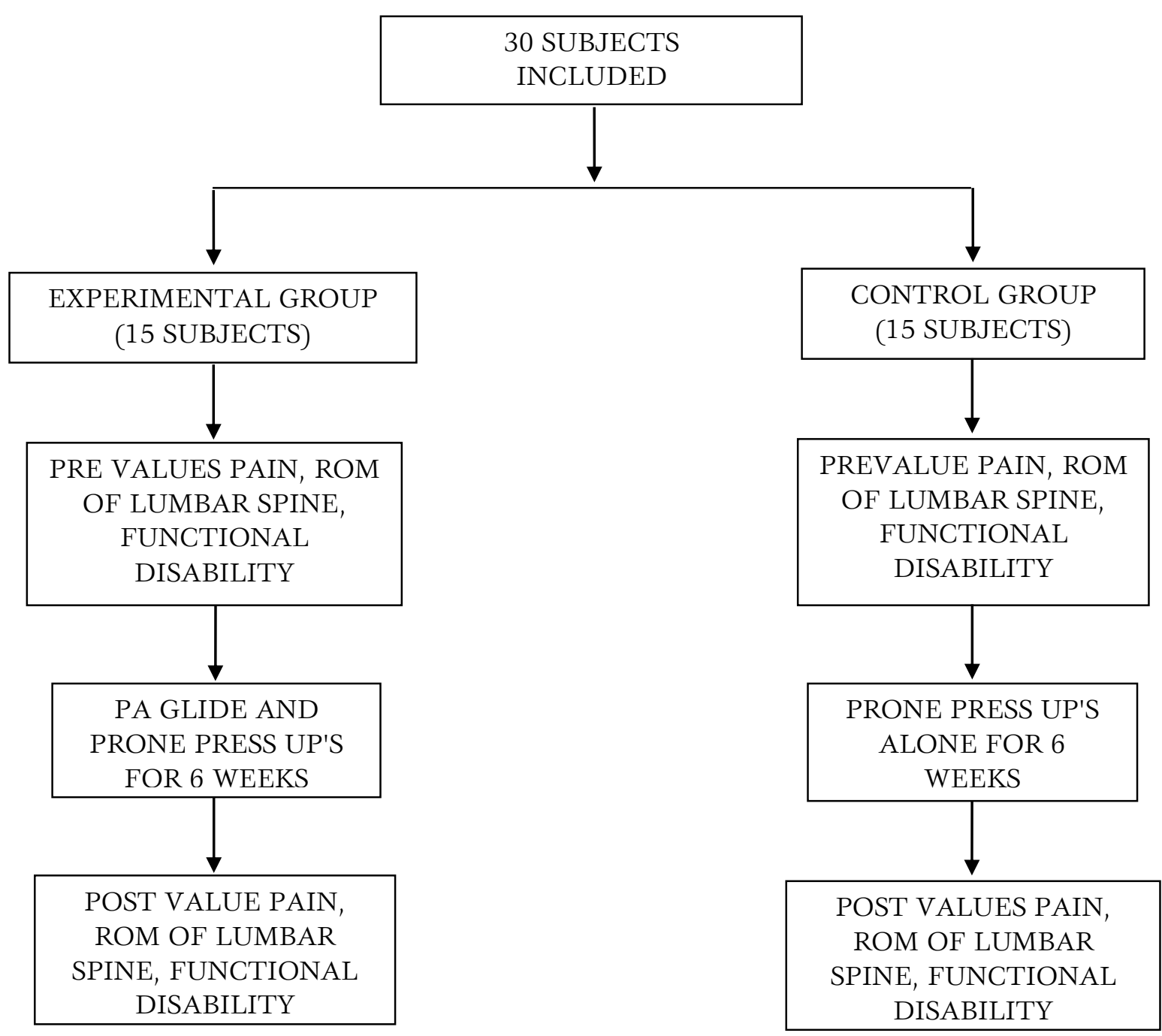

\section{INTERVENTION:}

For Group I mobilization was done with subject in prone position with their hands on either side of the treatment table. The direction of applied force was downwards and oscillations for 40 seconds were given for each lumbar vertebrae. Three bouts with 1 to $2 \mathrm{~Hz}$ of grade I Maitland mobilization was given if the patient can tolerate it continued to the highest amplitude tolerated without the reproduction of symptoms. The total time for the Posterior to Anterior lumbar mobilization intervention is approximately 10 minutes. ${ }^{12}$ After mobilization subjects will be asked to perform a prone press-up maneuver, the subject uses the arms to press the top half of the body upward into spinal extension, while the pelvis is allowed to sag with gravity and remain on the treatment table. The subject will be instructed to move from the prone position to maximum pain-free before the subject returns to the starting position. A total of 10 repetitions will be performed. The total time for the prone press-up exercise intervention is approximately 10 minutes. Treatment was given for 5 times a week for 6 weeks and 3 sets of 15 repetitions with rest interval off 30 seconds was included between the repetitions. For Group II Prone Press-up Exercises are advised. After the completion of intervention (at the end of 6 weeks) all the subjects' responses are recorded in the form of pain (VAS), lumbar extension ROM and functional disability (MODI questionnaire)

STATISTICAL METHODS: Statistical analysis has been carried out to analyze the significant impact of the treatment issued to the subjects of both control and experimental groups by using IBM SPSS Inc.20.0 version for this purpose the data was entered into Microsoft excels spreadsheet, tabulated and subjected to statistical analysis. 30 subjects 15 were randomised into control group and 15 were randomised into experimental group. All the 30 subjects completed entire protocol as defined by 6 weeks of treatment. The outcomes of the study were pain, lumbar spine extension ROM, functional disability. Statistical tools unpaired t-test has been applied for parameters in between groups and paired sample t-test for parameters within 
group. Descriptive measures like mean, standard deviation have been reported along with p-value.

RESULTS:

TABLE - 1: COMPARISON OF MEAN DIFFERENCE OF PRE EPOST VALUES OF PAIN AMONG EXPERIMENTAL AND CONTROL GROUPS:

\begin{tabular}{c|c|c|c|c|c|c|} 
Parameter & Group & N & Mean & SD & t-value & p-value \\
\multirow{2}{*}{ PAIN } & Experimental & 15 & 3.46 & 0.63 & 11.68 & 0.00 \\
\cline { 2 - 7 } & Control & 15 & 2.00 & 0.53 & & \\
\hline
\end{tabular}

GRAPH -1: GRAPHICAL REPRESENTATION OF POST MEAN DIFFERENCES OF PAIN BETWEEN BOTH GROUPS

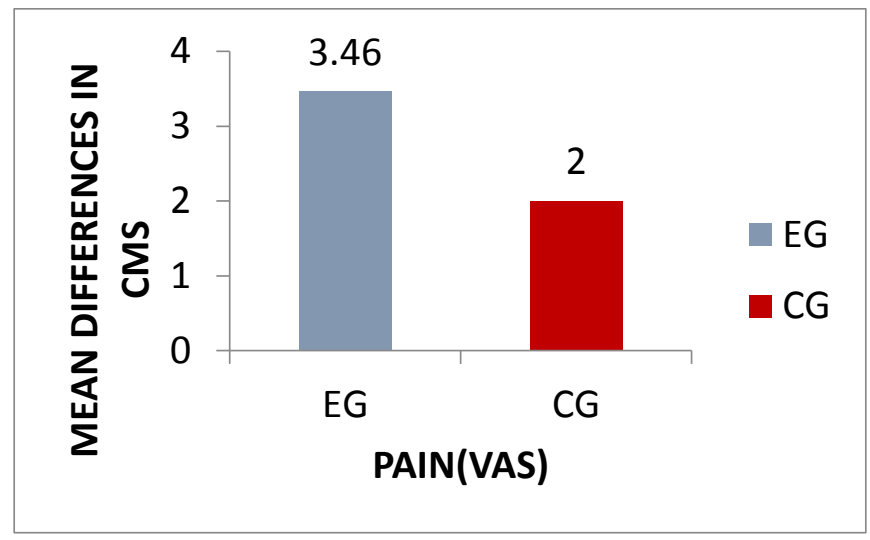

TABLE - 2: COMPARISON OF MEAN DIFFERENCE OF PREEPOST OF ROM OF LUMBAR SPINE AMONG EXPERIMENTAL AND CONTROL GROUPS:

\begin{tabular}{|c|c|c|c|c|c|c|}
\hline \multirow{2}{*}{ Parameter } & Group & N & Mean & SD & t-value & p-value \\
\cline { 1 - 7 } ROM OF LUMBAR SPINE & experimental & 15 & 1.06 & 0.41 & 5.33 & \multirow{2}{*}{0.00} \\
\cline { 2 - 7 } & Control & 15 & 0.66 & 0.67 & & \\
\hline
\end{tabular}

GRAPH - 3: GRAPHICAL REPRESENTATION OF POST MEAN DIFFERENCE VALUES OF ROM OF LUMBAR SPINE OF EXPERIMENTAL AND CONTROL GROUPS

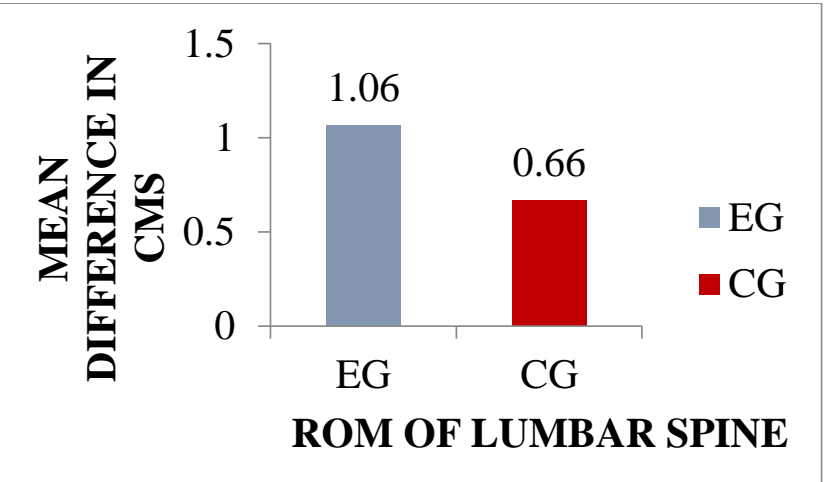

TABLE - 3: COMPARISON OF MEAN DIFFERENCE OF PREEPOST VALUES OF FUNCTIONAL DISABILITY AMONG EXPERIMENTAL AND CONTROL GROUPS:

\begin{tabular}{|c|c|c|c|c|c|c|}
\hline \multirow{2}{*}{ Parameter } & group & N & Mean & SD & t-value & p-value \\
\hline \multirow{2}{*}{ FUNCTIONAL DISABILITY } & experimental & 15 & 42.1 & 5.18 & 8.04 & 0.00 \\
\cline { 2 - 8 } & control & 15 & 27.33 & 4.86 & & \\
\hline
\end{tabular}




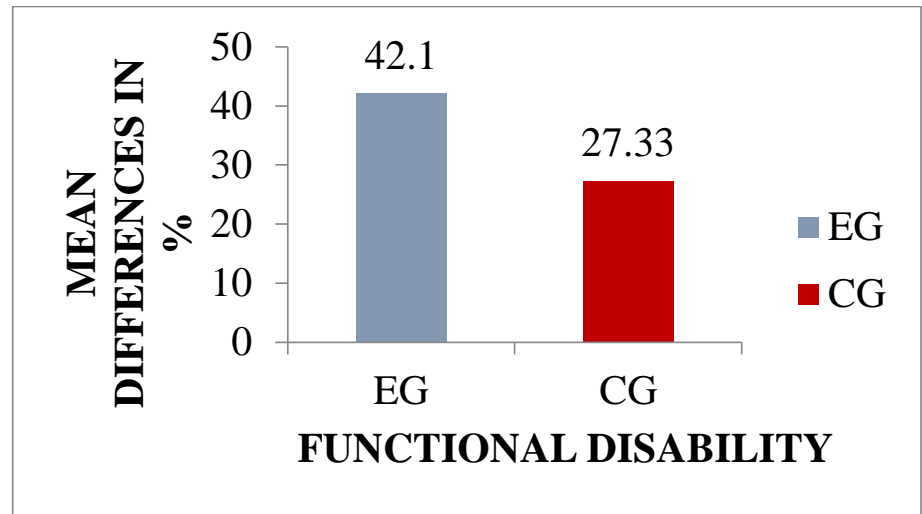

\section{DISCUSSION}

The age group of the participants was in between 35 to 50 years. Subjects above 50 years of age are prone for low back pain due to specific structural changes simply because of the process of aging. Hence subjects above 50 years of age have not been included in the study as the study involves nonspecific low back pain. Significant relief of pain was noted in both groups over sessions for 6weeks. When the intra-group mean values of visual analogue scale (VAS) were analyzed it was found statistically significant in both groups pre to post intervention; and post intervention at the end of 6 weeks, but when comparison was done intergroup, statistically significance difference was found between the two groups (EG $=63.36 \%$; $C G=36.63 \%)$ in relieving pain. Postero-anterior spinal mobilisation proved superior in terms of reduction of pain. In the present study reduction in pain level, as quantified by the VAS, with the application of both postero-anterior mobilisation and prone press-ups is consistent with the findings of previous studies indicating both the techniques reduced low back pain. In the present study, the intra-group mean values of lumbar extension range were analysed, it was found statistically significant in both groups at the end of 6 weeks, but when comparison was done intergroup, statistically significant difference was found between groups ( $E G=61.62 \% ; C G=38.37 \%$ ). Postero-anterior spinal mobilisation proved better in improving lumbar extension immediately post intervention as well as end of 6 weeks. Both mechanical and neurophysiologic mechanisms have been described to explain pain reduction and improved mobility following joint motion or mobilization, and it is conceivable that both mechanisms played a role in the findings of the present study. Passive motion has been reported to selectively stretch contracted tissues without damaging healthy adjacent tissues. ${ }^{13}$
Small-diameter sensory nerve fibres are likely activated, although this has not been demonstrated directly. The phenomenon of central facilitation is known to increase the receptive field of central neurons, enabling either sub threshold or innocuous stimuli access to central pain pathways. Spinal manipulation is also thought to affect reflex neural outputs to both muscle and visceral organs. Substantial evidence demonstrates that spinal manipulation evokes paraspinal muscle reflexes and alters motor neuron excitability

Twomey et al, demonstrated repetitive movements are thought to distribute synovial fluid over the articular cartilage and disk, resulting in less resistance to motion. ${ }^{14}$ With less resistance to motion, subjects may have felt free to move and thus may have experienced less pain

Chiradejnant et al reported a 36\% reduction in pain following two 1-minute bouts of spinal mobilization in subjects with nonspecific low back pain. ${ }^{13}$ Goodsell et al also studied the effects of PA mobilization on nonspecific low back pain and reported an average pain reduction of $33 \% .{ }^{15}$ The present study supports the work of McCollam and Benson, reported a $7.1 \%$ increase in lumbar extension, as measured with 2 fluid-based inclinometers on application of three 1 minute bout postero-anterior mobilisation at L3, L4 and L5. ${ }^{16}$

Another Cochrane Review of the Literature concluded that there is moderate evidence that spinal manipulative (SMT) / mobilization (MOB) is superior to general practitioner management for short-term pain reduction and that SMT offers similar pain relief to high-technology rehabilitative exercise in the short and long term. ${ }^{17}$

The Modified Oswestry Disability Index (MODI) Questionnaire is one of the most commonly used questionnaire for assessing disability in people with low back pain. The MODI has shown greater reliability and has sufficient width scale to reliably 
detect improvement or worsening in most subjects. For these reasons, the MODI appears to be the one of most commonly reported measure of health status in patients with low back pain.

In the present study, when the means of Modified Oswestry Disability Index Questionnaire (MODI) were analyzed intra groups, statistically significant improvement was found in both groups and has shown reduced MODI scores which represent an improvement in pain, range of motion and functional activities. When intergroup analysis was done, results have shown the statistically significant difference between group 1 and group 2 ( $E G=60.63 \%$; $C G=39.36 \%$ ), with group A showing better improvement in MODI scores. Thus it can be concluded that postero-anterior spinal mobilisation technique is more effective in improving physical function outcome in patients with non-specific low back pain.

\section{CONCLUSION}

In conclusion, the present randomized clinical trial provided evidence to support the use of manual therapy techniques, postero-anterior spinal mobilisation and prone press-ups in relieving pain, improving range of motion and reducing disability in subjects with non-specific low back pain. In addition, results supported that postero-anterior mobilisation was more effective than prone pressups in reducing pain and disability and improving lumbar extension in subjects with chronic nonspecific low back pain.

\section{Limitations:}

Small sample size, Subjects could not be followed up after the study, Duration of the study was short, and the strict inclusion criteria used in the present study limit the generalizability of the results to all low back pain populations, Activity level of subjects was not taken into consideration.

\section{RECOMMENDATIONS}

Studies with longer duration are recommended with longer follow-up period to assess long term benefits; Conduct the study with larger sample size, this study was confined to non-specific causes of low back pain. Future studies could be done taking up other causes, further studies are required to assess the effect of PA lumbar spine mobilization on pain, ROM and functional disability in male subjects with chronic nonspecific low back

\section{REFFERENCES}

1. Marras WS, Rangarajulu SL, Lavender SA. Trunk loading and expectation. Ergonomics. 1987; 30(3):551-562.
2. Burton AK. Battie MC. Gibbons L. et al. Lumbar disc degeneration and sagittal flexibility. J Spinal Disord.1996:9(5);418-424.

3. Sharma S.C, Singh R, Sharma A.K Mittal R; Incidence of low back pain in work age adults in rural North India. Indian J Med Sci.2003; 57(4): 145-147.

4. Krismer M, van Tulder M. Low back pain (nonspecific). Best Practice and Research: Clinical Rheumatology 2007; 21(1):77-91.

5. Akuthota V, Nadler SF. Core strengthening. Arch Phys Med Rehabil. 2004;85(3):86-92.

6. Samuel S Turek. Orthopaedic Principles and the application.4th Ed; 1977.

7. Porter, S. Dictionary of physiotherapy. $1^{\text {ST }}$ ed; 2005.

8. Maitland G D, Hengeveld. E, Banks K, and English K. eds. Maitland's Vertebral Manipulation. $7^{\mathrm{TH}}$ ed;2005.

9. McKenzie R A: The Lumbar Spine. Mechanical Diagnosis and Therapy. $1^{\mathrm{ST}} \mathrm{ed} ; 1981$.

10. Van Wijmen P M: "The management of recurrent low back pain." Modern manual therapy of the vertebral column. Ed. Grieve GP Ch 73:756-776, 1986.

11. Bigos S, Bowyer O, Braen G, Brown G, Deyo R, Haldeman S, John L, Hart D, Ernest W, Johnson M, Robert B, Keller M, Daniel K, Kido M. Acute low back problems in adults: assessment and treatment. Agency for Health Care Policy and Research, Clin Pract Guidel Quick Ref Guide Clin. 1994. pp. iii-iv. 1-25.

12. Maitland GD. Maitland's vertebral manipulation. 4th ed; 2007.

13. Chiradejnant A, Latimer J, Maher CG, Stepkovitch N. Does the choice of spinal level treated during Postero- anterior mobilization affect treatment outcome? Physiother Theory Pract. 2002; 18:165-174.

14. Twomey L, Taylor J. Exercises of spinal manipulation in treatment of low back pain. Spine 1995; 20: 615-619.

15. Goodsell M, Lee M, Latimer J. Short-term effects of lumbar posteroanterior mobilization in individuals with low-back pain. J Manipulative Physio Ther. 2000; 23(5):332-342.

16. McCollam RL, Benson C. Effects of Posteroanterior mobilization on lumbar extension and flexion. Journal of Manual and Manipulative Therapy.1993; 1(4):134-141.

17. Haas, M, Goldberg, B, Aickin, M, Ganger, B, Attwood, M. A practice-based study of patients with acute and chronic low back pain attending primary care and chiropractic physicians: twoweek to 48-month follow-up. J Manipulative Physiol ther. 2004; 27(3):160-169. 


\section{Citation}

L. V. S.Pravallika, C. Shanthi, \& K. Madhavi. (2014). EFFECTS OF POSTEROANTERIOR LUMBAR SPINE MOBILIZATIONS ON PAIN, ROM AND FUNCTIONAL DISABILITY IN FEMALE SUBJECTS WITH CHRONIC NONSPECIFIC LOW BACK PAIN. International Journal of Physiotherapy, 1(5), 279285. 